negative outcome a higher rate of Cesarean section, instrumental delivery $(\mathrm{OR}=1.96)$ and pathologic final delivery positions $(15.3 \%$ non-diabetes $/ 9.3 \%$ diabetes) can be observed. This all leads to a four times higher odds ratio of post-partus hospitalization $(\mathrm{OR}=3.70)$ and mandatory pre-partus therapy $(\mathrm{OR}=4.78)$ of the diabetes affected group.

Conclusions Newborns of mothers with gestational diabetes demonstrate a fetopathia diabetica with makrosomi and immaturity which leads to higher rate of birth complications and postpartus hospitalisation of almost every second newborn. An increased risk for mothers and their children exists in case of gestational diabetes.

\section{MALE VERY-LOW-BIRTH-WEIGHT AND VERY-LOW- GESTATIONAL-AGE INFANTS HAVE ADVERSE PERINATAL OUTCOMES}

doi:10.1136/archdischild-2012-302724.0985

${ }^{1} \mathrm{~A}$ Valls-i-Soler, ${ }^{2} \mathrm{~A}$ Azpeitia, European Neonatal Network (EuroNeoNet). ${ }^{1}$ Cruces University Hospital; ${ }^{2}$ BIOEF, Barakaldo, Spain

Background It's generally accepted that male Very Low Birth Weight (VLBW)/Very Low Gestational Age (VLGA) infants have a higher neonatal mortality rate (NMR), and this has been questioned recently (Arch Dis Child Fetal-Neonatal Ed. 2009; 94:F140-3).

Aim To determine if male gender has an adverse effect on NMR and early morbidity in VLBW/VLGA infants.

Methods Perinatal risk/protective factors, early neonatal complications and NMR were compared between male and female VLBW/ VLGA infants admitted from 2006 to 2010 in 174 EuroNeoNet NICUs from 17 European countries $(\mathrm{N}=28,035)$. Independent comparisons using non-parametric tests and logistic regression models were performed to predict adjusted NMR. Crude and adjusted odds ratios (OR) were used to determine perinatal and early neonatal associations.

Results Male infants (52.3\%) had a lower GA and higher BW than females. Crude NMR was higher in males (14 vs. $11.6 \%$; OR=1.24 95\%CI: (1.16-1.33)) as were most perinatal and early neonatal risk factors (vaginal delivery, low Apgar scores, resuscitation, RDS and need for surfactant therapy and early-onset sepsis), as well as major adverse neonatal outcomes (pneumothorax, late-onset sepsis, BPD, $\mathrm{IVH}_{3-4}, \mathrm{PVL}$ and $\mathrm{ROP}_{3-4}$ ). These differences in NMR and complications remained after adjusting for GA and/or BW alone and by perinatal differentiating factors for gender: BW, GA, 1-min Apgar score and multiple pregnancy (NM: OR=1.32 95\%CI: 1.21-1.44).

Conclusion Male infants of VLBW/VLGA have a higher adjusted NMR and an increased rate of adverse neonatal outcomes.

Acknowledgements We thank patients and NICUs participating NICU's. EuroNeoNet is supported by the DGSANCO (EuroNeoStat project No 2008/1311).

\section{A COMPARISON BETWEEN EFFECTS OF VAGINAL MISOPROSTOL AND OXYTOCIN IN SUCCESSFUL INDUCTION OF LABOR: A DOUBLE BLIND CLINICAL TRIAL}

doi:10.1136/archdischild-2012-302724.0986

M Bakhtiyari. Tehran University of Medical Sciences, Tehran, Iran

Aim and Background: Labor induction for Prepare the cervix to active phase of labor, is now one of the most common methods in the field of Obstetrics and Gynecology. This study was conducted to compare the effects of misoprostol and oxytocin in BoAli Hospital, Tehran in 2011.

Method This study was a double-blind clinical trial by recruitments of 60 primiparous women that has been referred to BoAli hospital in Tehran in 2011. Individuals were divided into two groups by random allocation. For induction of labor, misoprostol and oxytocin were prescribe in individuals who candidate for pregnancy termination by four hours intervals. For Analysis of data, t test, analysis of variance with repeated measurements, linear regression and descriptive statistics were used.

Results Mean age and standard deviation in intervention group was $24.07 \pm 3.96$ and in control group equal to $24.13 \pm 3.97$. Kind of treatment variable, cause of admission and hypertension history shown a significant association with increased or decreased time to achieve regular and suitable contractions for delivery $(p<0.05)$. Results of $t$ test in two groups of intervention and control shown that until three hours after receive Assigned treatment, there was no statistical significancy between two groups of study, But then we are clearly seeing the better impact of misoprostol drug in the intervention group ( $\mathrm{p}=0.014)$.

Conclusion Using misoprostol in labor induction in order to reducing the Time to reach the short and effective contraction is more effective Instead of oxytocin and it can be a good alternative.

\section{EMIPEDIOLOGY AND MORBIDITY OF LATE PRETERM NEONATES IN CORRELATION WITH MATERNAL RISK FACTORS IN HESSEN, GERMANY}

doi:10.1136/archdischild-2012-302724.0987

'D Faas, 'M Vajz, ${ }^{2} \mathrm{U}$ Hennewig, ${ }^{3} \mathrm{~B}$ Misselwitz, ${ }^{4} \mathrm{~F}$ Oehmke, ${ }^{5} \mathrm{M}$ Heckmann. ${ }^{1}$ General Pediatrics and Neonatology, Justus-Liebig-University Giessen, Giessen; 2Pediatric Hematology and Oncology, Johannes Gutenberg University of Mainz, Mainz; ${ }^{3}$ nstitut for Quality Assurance Hessen, Eschborn; " Department of Obstetrics and Gynecology, Justus-Liebig-University Giessen, Giessen; ${ }^{5}$ Neonatology and Pediatric Intensive Care, Ernst Moritz Arndt University Greifswald, Greifswald, Germany

Short and long term morbidity in late preterm neonates (LP) and their correlation to maternal risk factors are relevant as shown in multiple studies in North America and European countries. Epidemiological studies showed an increase in LP birth over time. Corresponding data for Germany have not yet been published. Our goal was to evaluate the epidemiology of LP over a 7 year period and the correlation to potential maternal risk factors in Hessen, Germany.

Data was collected from the perinatal und neonatal quality assurance in Hessen form 2001 to 2007. For some calculations the two data sets were merged. Overall the data sets of 360.000 births and 44.000 neonatal hospitalizations were available, which is about $7.5 \%$ of all births during that period in Germany.

There is no increase in LP births from 2001 to 2007 in Hessen. Neonatal short term morbidity of LP is within the expected range and significantly higher than in term neonates. The following maternal risk factors were more predominant in LP: placental insufficiency, gestational diabetes, obesity, arterial hypertension, artificial reproduction techniques, caesarian section in previous pregnancy, ethnic background and multiple births. Maternal morbidity has an influence on the short term morbidity of LP.

There has been no increase in late preterm births in Hessen Germany 2001-2007. Several maternal risk factors for late preterm births can be identified. These results could be used to optimize management of mothers at risk to reduce the number of late preterm births and neonatal morbidity.

\section{EFFECTS OF IRON SUPPLEMENTATION OF LOW BIRTH WEIGHT INFANTS ON COGNITION AND BEHAVIOR AT 3 YEARS}

doi:10.1136/archdischild-2012-302724.0988

'S Berglund, ${ }^{2 B}$ Westrup, ${ }^{3} \mathrm{~B}$ Hägglöf, ${ }^{1} \mathrm{O}$ Hernell, ${ }^{1} \mathrm{M}$ Domellöf. ${ }^{1}$ Clinical Science, Pediatrics, Umeå University, Umeå; ${ }^{2}$ Women and Child Health, Neonatology, Karolinska Institute, Stockholm: ${ }^{3}$ Clinical Sciences, Child and Adolescent Psychiatry, Umeå University, Umeå, Sweden 
Objective Low birth weight infants (LBW) are at increased risk of cognitive and behavioral problems and at risk of iron deficiency (ID) which is associated with impaired neurodevelopment. We hypothesized that iron supplementation of LBW infants would improve cognitive scores and reduce behavioral problems.

Patients and methods: In a randomized controlled trial, 285 marginally LBW (2000-2500g) infants received 0 , 1, or $2 \mathrm{mg} / \mathrm{kg} /$ day of iron supplements from six weeks to six months of age. At 3.5 years of age these infants and 95 normal birth weight controls were assessed with a psychometric test (WIIPSI-III) and a questionnaire of behavioral problems (CBCL).

Results There were no significant differences in IQ between the LBW-groups, nor compared to controls. Mean (SD) full-scale IO was 105.2 (14.5), 104.2 (14.7), and 104.5 (12.7) in the placebo, $1 \mathrm{mg}$, and 2 mg-group respectively ( $p=0.924)$. The prevalence of children with CBCL-scores above the US subclinical cut-off was $12.7 \%, 2.9 \%$, $2.7 \%$, and $3.2 \%$ in the placebo, $1 \mathrm{mg}, 2 \mathrm{mg}$, and control-group respectively. Relative risks ( $95 \% \mathrm{CI}$ ) for behavioral problems vs. controls were 4.01 (1.13-14.29) in the placebo-group. In a logistic regression model, adjusted for confounders, the odds ratio (95\% CI) for CBCL score above US subclinical cut-off in placebo-treated children was 4.5 (1.3-15.8) compared to iron supplemented children $(p=0.019)$.

Conclusions Early iron supplementation of marginally LBW infants does not affect cognitive functions at 3.5 years of age but significantly reduces the prevalence of behavioral problems. The study suggests a causal relation between infant ID and later behavioral problems.

\section{A RETROSPECTIVE COHORT STUDY TO ASSESS THE ASSOCIATION BETWEEN OUTDOOR AIR QUALITY AND LOW BIRTH WEIGHT}

doi:10.1136/archdischild-2012-302724.0989

${ }^{1} \mathrm{MC}$ Ribeiro, ${ }^{2} \mathrm{E}$ Llop, ${ }^{3} \mathrm{C}$ Branquinho, ${ }^{4} \mathrm{CM}$ Dias, ${ }^{5} \mathrm{~A}$ Tavares, ${ }^{6} \mathrm{~F}$ Santos, ${ }^{1} \mathrm{~A}$ Soares, ${ }^{1} M J$ Pereira. ${ }^{1}$ Cerena/DECivil, Instituto Superior Técnico; Universidade Técnica de Lisboa, Lisbon, Portugal; 'Biologia Vegetal-Botánica, Universitat de Barcelona, Barcelona, Spain; ${ }^{3}$ Ecologia e Biologia Vegetal, Universidade de Lisboa; Faculdade de Ciencias; ${ }^{4}$ Epidemiologia, Instituto Nacional de Saúde Ricardo Jorge; ${ }^{5}$ Administração Regional de Saúde Lisboa e Vale do Tejo, Direção Geral de Saúde, Lisbon; ${ }^{\circ}$ Administração Regional de Saúde Alentejo, Direção Geral de Saúde, Sines, Portugal

Introduction Low birth weight (LBW) is defined by the World Health Organization as weight at birth of less than 2500 g. Epidemiological observations suggest that LBW contributes to a range of poor infant's health outcomes. Other studies suggest that an increase of outdoor air pollution levels may increase the incidence of LBW. This article presents results from a semi-ecological analysis of association between outdoor air quality and LBW in a cohort of mothers participating in Gestão Integrada Saúde e Ambiente (GISA) project, in Alentejo Litoral region (Portugal).

Materials Individual data on birth weight, residence, demographic, social and clinical covariates were collected by questionnaire from mothers ( $n=1393$ ) participating on GISA project. Air quality data was collected with a lichen diversity biomonitoring program measured at spatiality distributed sampling sites $(n=84)$.

Methods Lichen biomonitoring was used to derive a continuous metric of outdoor air quality exposure. Geostatistical simulation was applied to lichen diversity data to derive equally probable maps of air quality with different exposure scenarios for each pregnant, to gain insight into exposure distribution and exposure uncertainty. Generalized linear models were used to predict the odds of LBW.

Results Factors found significantly $(p<0.05)$ associated to LBW: smoking habits, prenatal surveillance, body mass index, intrauterine growth, weight gain during pregnancy, previous LBW. Air quality was not associated to LBW (odds, 1.001; confidence interval 95\%, 0.998-1.006).
Conclusions Air quality was not associated to LBW. Factors found to be significantly associated with LBW are in line with scientific knowledge.

\section{ETHNIC DIFFERENCES IN NEONATAL BODY COMPOSITION AND THE IMPACT OF PARENTAL SIZE. A POPULATION- BASED COHORT STUDY}

doi:10.1136/archdischild-2012-302724.0990

${ }^{1,2,3} \mathrm{~L}$ Sletner, ${ }^{1,3 \mathrm{~B}}$ Nakstad, ${ }^{2} \mathrm{~S}$ Vangen, ${ }^{3,4} \mathrm{~K}$ Mørkrid, ${ }^{3,4} \mathrm{~K}$ Birkeland, ${ }^{5} \mathrm{AK}$ Jenum. ${ }^{1} \mathrm{Child}$ and Adolescents Medicine, Akershus University Hospital, Lørenskog; ${ }^{2}$ Norwegian Resource Centre for Women's Health, Oslo University Hospital; ${ }^{3}$ nstitute of Clinical Medicine, University of Oslo; ${ }^{4} \mathrm{Dep}$. of Endocrinology, Oslo University Hospital; ${ }^{5} / n s t i t u t e$ of Health and Society, University of Oslo, Oslo, Norway

Background and Aim Neonatal size, and especially a relative "thinness", is associated with future risk of obesity and type 2 diabetes. The aim was to explore ethnic differences in neonatal body composition in a multi-ethnic population, and associations with parental anthropometrics and parity.

Methods A population-based cohort study of healthy pregnant women living in Oslo, Norway. Maternal data were collected in early pregnancy and neonatal weight, length, circumferences and skin folds after birth. Individual z-scores were calculated (stratified by gender and gestational age) with Western Europeans (WE) as reference. A general linear model was used to assess associations with parental anthropometry and parity. Crude and adjusted mean z-scores for neonates with origin from low- and middle income countries (LAMIC), with WE as reference, is presented.

Results 511 healthy, term neonates were included. LAMIC parents $(n=282)$ were shorter than WE $(n=229)$, had similar BMI but more maternal subcutaneous fat. Among the LAMIC neonates abdominal circumference differed the most from WE (mean z-score: $-0.57 \mathrm{SD}$ (95\% CI:-0.69 to -0.44)) Neonatal skin folds were also smaller in the immigrant group $(-0.29$ ( -0.40 to -0.17$)$. Length, however, was not significantly smaller $(-0.21(-0.35$ to -0.07$))$. The observed ethnic differences were reduced when adjusting for parental body composition, but abdominal circumference remained significantly smaller $(-0.37$ ( -0.53 to -0.21$)$ ).

Conclusions There were marked differences in neonatal body composition between neonates born by ethnic WE women and women with ethnic origin from LAMIC countries. Abdominal circumference was still smaller after adjustment for parental body composition.

\section{NONINVASIVE VENTILATION IN CHILDREN, CAN WE PREDICT ITS FAILURE?}

doi:10.1136/archdischild-2012-302724.0991

P García Soler, MJ Vicente Martín, A Morales Martínez, JM González Gómez, G Milano Manso. Pediatric Intensive Care Unit, Hospital Regional Universitario Materno Infantil Carlos Haya, Málaga, Spain

Background and Aims Noninvasive ventilation (NIV) constitutes an alternative treatment for pediatric acute respiratory failure. However, tracheal intubation should not be delayed when considered necessary. Our main objective is to identify success/failure prognostic signs of NIV and determination of NIV characteristics. Methods Noncontrolled, observational study in a PICU in a university hospital. All cases of NIV from June 2010 and March 2012 were included. Failure of NIV was defined as the requirement of endotracheal intubation at any time. The major characteristics of patients who were intubated were compared with the characteristics of those who were not after a trial of NIV. Predictive factors for failure of NIV were analysed by multivariate analysis.

Results 126 cases were included (60.3\% males), mean age $25.3 \pm 40.5$ months and mean weight $11 \pm 13 \mathrm{~kg}$. There was $57.9 \%$ of type II 